RANDOMISED STUDY ON THE THERAPEUTIC EFFICACY OF ADDED TREATMENT WITH KANLI GRANULE IN PATIENTS WITH CHRONIC HEART FAILURE

doi:10.1136/hrt.2010.208967.619

${ }^{1}$ Meixian Jiang, ${ }^{2} \mathrm{Li} \mathrm{Ma},{ }^{1}$ Xiaofen Ruan, ${ }^{1} \mathrm{Yan} \mathrm{Xu} .{ }^{1}$ Shanghai Shuguang Hospital, Shanghai, China; ${ }^{2}$ Xin Jiang Municipality Hospital, Wulumuqi, China

Aims To evaluate the efficacy of added treatment with Kanli granule in the patients with chronic heart failure (CHF).

Method 124 patients with CHF were involved in this study, and were divided into group TCM and the Control (both with 62 cases). All the patients received conventional therapy according to the guidelines of AHA/ACC for 24 weeks, and KanLi granule (containing 10 herbal medicines) $40 \mathrm{~g} \mathrm{day}^{-1}$ for 24 weeks in addition only in group TCM. The syndrome integration (TCM), $6 \mathrm{MWD}$, LHFO score, curative efficacy, and withdrawal rates of diuretics and digoxin were observed at 0th, 4th, 8th, 12th and 24th week of treating period, LVEF assessed by echocardiography before and after treatment, the annual mortality and re-hospitalisation due to acute aggravation of heart failure (HF) were recorded as well.

Results The follow-up was accomplished in 109 patients (55 in group TCM, 54 in Control). 3.1. Syndrome integration significantly decreased both in 2 groups since 8th week of treatment $(p<0.01)$, with the advantage in group TCM $(p<0.05$ or $p<0.01)$. The curative efficacy of syndrome integration had been raised since 4 th week in both two groups $(p<0.01)$, and more excellent in group TCM (since 4 th week, $\mathrm{F}=10.703, \mathrm{p}<0.01)$. Therapeutic efficacy on NYHA grade increased since 8th week in group TCM $(\mathrm{p}<0.01)$, and the Control, since 12th week $(p<0.05)$, with advantage in group TCM too (since 8 th week, $p<0.001)$. The LVEF increased in group TCM after treatment $(p<0.05)$, but in the Control, no obvious change. 3.2. There had been significant increase of $6 \mathrm{MWD}$ and decrease of LHFQ score in both group since 4 th week $(p<0.01)$, with longer 6MWD (since 8th week, $\mathrm{F}=13.324, \mathrm{p}<0.01$ ) and the lower LHFQ scores (since 4th week, $\mathrm{F}=44.78, \mathrm{p}<0.01$ ) in group TCM. 3.3. The withdraw rate of diuretics (since 8th week, $\mathrm{p}<0.01$ ) and of digoxin (since 12th week, $\mathrm{p}<0.01$ ) in group TCM had increased more significantly than that in the group Control. 3.4. The annual re-hospitalisation rate due to acute aggravation of HF in group TCM was lower than that in the Control $(p<0.05)$, so as the annual death $(4.84 \%$ vs $11.29 \%)$, but without statistic difference because of the small case sample.

Conclusion 4.1. The patients with CHF received added treatment with KanLi granule may obtain more benefits as follows: increasing curative efficacy and LVEF, improving exercise tolerance and life quality, reducing use of diuretics and digoxin, reducing annual rehospitalisation due to acute aggravation, and perhaps annual death as well. 4.2. The multiple advantages of the added therapy with TCM had presented earlier since 4th and lately since 12 th week of treating period, so the treating period at least will be 12 weeks. 4.3. To evaluate the efficacy of combination therapy with TCM and conventional treatment on patients with CHF, the evaluating system should include short-term therapeutic efficacy (TCM syndrome integration and NYHA grade), laboratory indices (LVEF, $\mathrm{BNP})$, quality of life (6MWD and LHFQ), the diuretics and digoxin withdrawal rate and long-term indices (annual re-hospitalisation and mortality), then the annual medical cost of CHF if possible.

\section{Q0620 APACHE-II SCORING SYSTEM IS USED IN CRITICALLY ILL PATIENTS WITH CARDIOVASCULAR DISEASE}

doi:10.1136/hrt.2010.208967.620

Yu Wang, Jun Zhu, Huiqong Tan, Yan Zhang. Fuwai Cardiovascular Hospital

Objective To investigate the acute physiology and chronic health evaluation (APACHE)-II for evaluating the severity of the illness in patients with cardiovascular disease and the ability to predict prognosis

Methods Selected 1439 patients, who were admitted into the ICU for treatment in Fuwai Hospital from 2003 to 2008, calculated each patient's APACHE-II score and the risk of death ( $\mathrm{R}$ value). In accordance with the APACHE-II scores $(<10,10-20,>20)$ and the type of ICU common diseases (congenital heart disease; coronary heart disease; valvular heart disease; cardiomyopathy; pulmonary heart disease), all the patients were divided into groups to analyse the consistency of actual mortality and predicted mortality and the relationship between actual mortality and APACHE-II scores. Compared the overall scores and each section of the non-survival group and survival group of APACHE-II scores.

Results 1439 cases of patients, the actual death case is 122, male 77, female 45 . Predicted mortality and actual mortality both are related to APACHE-II scores, they increased along with APACHE-II scores, the mortality of $>20$ group is $45.5 \%$. $<10$ group APACHE-II score, non-survival group $(7.38 \pm 1.389)$ and survival group $(7.44 \pm 1.464)$ were not significantly different ( $p>0.05$ ). In 10 to 20 group, $>20$ group and the overall, APACHE-II score of non-survival was significantly higher than the survival group $(p<0.05)$. APACHE-II method to the mortality rate is expected to determine the individual prognosis, area under the ROC curve is 0.689 , low diagnostic value. Compared actual mortality and predicted mortality, divided by score, only $>20$ group predicted mortality is in the actual mortality rate $95 \% \mathrm{CI}, 10$ to 20 group, $>20$ group, there are significant differences between the predicted mortality and actual mortality. Divided by the disease, only the forecasting mortality of pulmonary heart disease group is in the actual mortality $95 \%$ CI. In remaining groups, there is significant difference.

Conclusion APACHE-II scores is related to the disease critical levels, the higher the score the higher the risk of death, a significant increase in mortality in $>20$ groups, clinicians should arouse great attention. In the high score ( $>20$ points), APACHE-II predicted mortality and actual mortality are similar. The prognosis of this part patient with cardiovascular disease have a certain value. But on the whole, APACHE-II predicted mortality for cardiovascular disease is not very well. Particularly in low scores ( $<10$ points, $10-20$ points), predicted mortality and actual mortality were significantly different, and in $<10$ group APACHE-II scores of the non-survival group and survival group were not significantly different, which the APACHEII score is not related to death in $<10$ group. In conclusion, APACHE-II scores indeed, to some extent, has a rough evaluation of the critical level of cardiovascular disease, especially in the high score section, but for mortality prediction, the value of their diagnosis is low.

\section{e0621 THE EFFECT OF DANHONG INJECTION ON THE PLASMA LEVELS OF BRAIN NATRARETIC PEPTIDES AND C-RESPONSE PROTEIN IN PATIENTS WITH UNSTABLE ANGINA}

doi:10.1136/hrt.2010.208967.62

Han Ling, Chen Jin-Liang, Liu Zhi-Ming, Chen Hong-Tao, Miao Yong-Guo. Department of Cardiology, Handan Peoples's Hospital, Hebei province, Hebei Handan, China

Objective To observe the curative effect and change of plasma levels of brain natriuretic peptides (BNP) and C-response protein (CRP) in patients with unstable angina.

Methods 118 patients with unstable angina were divided randomly into two groups, one was control group treated with regular treatment, and another was treatment group treated with danhong injection on the basis of above regular treatment. All the patients were followed up for fourteen days and observed the changes of the clinical symptoms, change of plasma levels of BNP and CRP. 